Short communication

\title{
Continuous use of textured insole improve plantar sensation and stride length of people with Parkinson's disease: A pilot study
}

\author{
Ellen Lirani-Silva ${ }^{\mathrm{a}, *}$, Rodrigo Vitório ${ }^{\mathrm{a}}$, Fabio Augusto Barbieri ${ }^{\mathrm{b}}$, Diego Orcioli-Silva ${ }^{\mathrm{a}}$, \\ Lucas Simieli ${ }^{\mathrm{b}}$, Lilian Teresa Bucken Gobbi ${ }^{\mathrm{a}}$ \\ a São Paulo State University (UNESP), Bioscience Institute, Campus Rio Claro, 1515 24-A Avenue, Bela Vista, Rio Claro, São Paulo State, 13506-900, Brazil \\ b São Paulo State University (UNESP), Faculty of Science, Campus Bauru, 14 Luís Edmundo Carrijo Coube Avenue, Vargem Limpa, Bauru, São Paulo State, 17033-360, \\ Brazil
}

\section{A R T I C L E I N F O}

\section{Keywords:}

Parkinson's disease

Textured insoles

Gait

Plantar sensation

Continuous use

\begin{abstract}
A B S T R A C T
Findings involving the acute benefits of textured insoles on gait in people with Parkinson's disease (PD) are still controversial. To our best acknowledge, the continuous use of textured insoles on gait in people with PD has not been investigated yet. The aim of this pilot study was to obtain preliminary data of the effects of textured insoles on gait and plantar sensation in people with PD after one-week intervention and one-week follow-up period. Nineteen patients with PD were distributed into two groups: experimental group and control group. Initially, the plantar sensation was assessed through Semmes-Weinstein Monofilaments. Then, participants performed 5 trials of walking (without insoles) at a self-selected speed. Gait data were collected using an optoelectronic system. Plantar sensation and gait assessments were repeated in three moments: before and after one-week wearing the group-specific insoles, and after one week wearing conventional insoles (follow-up). The textured insole had half-sphere elevations placed in the distal phalanx of the hallux, heads of metatarsophalangeal joints and heel. Results revealed that the use of textured insole for one week improved plantar sensation and stride length. However, only benefits on plantar sensation were maintained after the follow-up period. Our results suggest that the continuous use of textured insoles for one week benefits plantar sensation and gait in patients with PD. These results support the hypothesis that enhanced somatosensory feedback to the sensory system result in an improved motor output of gait.
\end{abstract}

\section{Introduction}

Gait impairments in Parkinson's disease (PD) are, at least in part, associated to somatosensory deficits such as decreased plantar sensation of the feet [1]. Increasing evidence suggest that augmented stimulation of the plantar surface, by wearing textured insoles, benefit gait in both healthy and clinical populations [2]. However, the few existing studies involving people with PD found controversial results. While Jenkins et al. observed acute benefit [3], our group observed no change when people with PD wore textured insoles [4]. It's important to highlight that Jenkins et al. found a trial by trial benefit on singlelimb support time and those benefits were more pronounced at the last trials. These results suggest that a long-term exposure to textured insoles may cause more pronounced benefits to gait. However, the effects of long-term use of textured insoles on gait have not been investigated yet.

The aim of this pilot study was to investigate the effects of continuous use of textured insoles on gait and plantar sensation of people with PD. We also investigated if potential effects are maintained after a follow-up period.

\section{Methods}

\subsection{Participants}

Nineteen patients with idiopathic PD were recruited according to the following inclusion criteria: stage 1 to 3 on the Hoehn and Yahr Scale (H \& Y [5]), independent walking and in regular use of PD-specific medication. Patients were randomly distributed in two groups: i) G1: textured insole $(n=10)$; ii) G2: conventional insole (control group, $\mathrm{n}=9$ ). The randomization procedure was carried out by draw. The exclusion criteria were: cognitive impairment based on suggestions of Brucki et al. for utilization of the Mini Mental State Examination (MMSE) in Brazil [6], comorbidities involving serious decrease in

\footnotetext{
* Corresponding author at: São Paulo State University (UNESP), Bioscience Institute, Physical Education Department, campus Rio Claro, Posture and Gait Studies Laboratory (LEPLO), Av. 24-A, 1515, Bela Vista, Rio Claro, São Paulo State, 13506-900, Brazil.

E-mail address: ellenlirani@gmail.com (E. Lirani-Silva).
} 
plantar sensation (e.g. diabetes) or other neurodegenerative pathology than PD. Participants provided written informed consent before enrolment in the study, which was approved by local Research Ethics Committee (\#3590/2010). All participants were assessed $1 \mathrm{~h}$ after taking PD-specific medication.

\subsection{Assessments}

The Unified Parkinson's Disease Rating Scale (UPDRS [7]) and the $\mathrm{H} \& \mathrm{Y}$ were applied to assess motor impairment and disease stage, respectively. The global cognitive function was assessed by the MMSE [6].

Plantar sensation was assessed before gait assessments using Semmes-Weinstein Monofilaments [8] while participants were laid in supine position. The sites of the sole of the foot examined were: 1st, 3rd and 5th metatarsal head and distal phalanx, and medial portion of the heel for right and left foot. The range score of each site was 1 to 7 , the sum of the scores of each site was calculated, and the average of left and right feet was considered for analysis. Higher scores represented greater deficits in plantar sensation [4].

For gait assessment, participants walked at a self-selected speed on an 8-m long pathway. Five trials were performed without insoles to prevent results related to immediate effects. Kinematic data were recorded using an optoelectronic tridimensional system (OPTOTRAK Certus ", Nothern Digital Inc.) at a frequency of $100 \mathrm{~Hz}$. Four infrared markers were attached at the participant's feet: 5th right and 1st left metatarsal joints and lateral face of the right calcaneus and medial face of the left calcaneus. The dependent variables included: stride length, duration and velocity, cadence, step width, stance phase duration and percentage of double-support phase duration [4].

Plantar sensation and gait of both groups were assessed in three moments: i) before (Pre-test) and; ii) after one week wearing the groupspecific insoles (Post-test); iii) after one week wearing conventional insoles (follow-up).

\subsection{Insole protocol intervention}

After the pre-test, participants wore group-specific insoles (textured/conventional) during one week and conventional insoles in the following week. The two insoles were made with the same material in the basis ( $2 \mathrm{~mm}$ high; medium density) and the size was customized to each participant [4]. Fig. 1 illustrates the textured insole. Participants were instructed to wear the insoles inside conventional shoes without changes in everyday habits. Also, to control the use of the insoles, participants were instructed to use a pedometer (SW-200, Yamax Corp).

\subsection{Statistical analysis}

Demographic and clinical data were compared through Student $t$ test for between-group comparisons. Two-way ANOVAs (Group X Test) were carried out to compare pedometer data (number of steps in one week), plantar sensation and kinematic variables. Post-hoc tests were used to localize differences where appropriate. Bonferroni correction

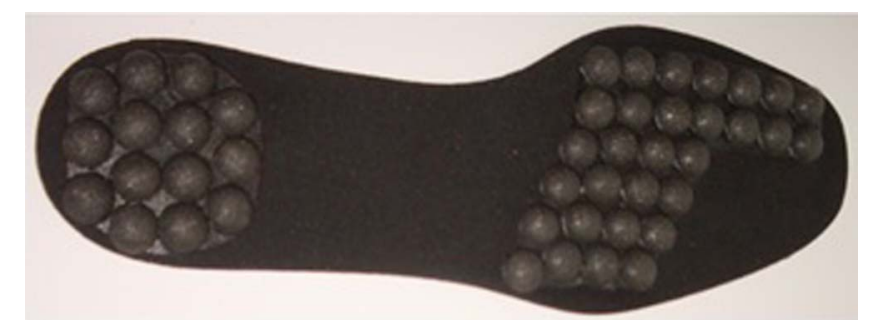

Fig. 1. Textured insole with half-sphere elevations. The textured insole had half-sphere elevations ( $9 \mathrm{~mm}$ of diameter), placed in the distal phalanx of the hallux, heads of metatarsophalangeal joints and heel.
Table 1

Demographic and clinical characteristics of patients at groups G1 (textured insole) and G2 (control insole).

\begin{tabular}{|c|c|c|c|}
\hline & $\mathrm{G} 1(\mathrm{n}=10)$ & $\mathrm{G} 2(\mathrm{n}=9)$ & $P$-value \\
\hline Age (years) & $70.4 \pm 6.87$ & $72 \pm 6.2$ & 0.602 \\
\hline Height $(\mathrm{cm})$ & $159.2 \pm 11$ & $161.3 \pm 6$ & 0.614 \\
\hline Weight (Kg) & $65 \pm 14.5$ & $66.5 \pm 7.42$ & 0.768 \\
\hline MMSE (score) & $27.6 \pm 2.11$ & $28 \pm 2.6$ & 0.716 \\
\hline H \& Y (stage) & $2 \pm 0.5$ & $1.9 \pm 0.4$ & 0.595 \\
\hline UPDRS III (score) & $29.5 \pm 6.05$ & $24.7 \pm 7.5$ & 0.138 \\
\hline UPDRS total (score) & $48.6 \pm 10.92$ & $41.5 \pm 11.7$ & 0.185 \\
\hline UPDRS - Gait \& Posture (score) & $4.60 \pm 3.20$ & $3.89 \pm 2.37$ & 0.593 \\
\hline Patients taking L-dopa (number) ${ }^{£}$ & 9 & 8 & 0.937 \\
\hline
\end{tabular}

Note: values are mean \pm SD, MMSE: Mini Mental State Examination, H \& Y: Hoehn and Yahr scale, UPDRS: Unified Parkinson's Disease Rating Scale, UPDRS - Gait \& Posture: gait and posture score based on sum of items 13, 14, 15 (UPDRS II) and items 29 and 30 (UPDRS III), ${ }^{£}$ Chi-squared analysis.

was applied for multiple comparisons. $P$ value $\leq 0.05$ was considered statistically significant.

\section{Results}

All participants completed the entire study protocol. The participants reported no major discomfort related to the insoles. There were no significant differences between groups for all demographic and clinical data (Table 1). A significant interaction between factors (Group $\mathrm{X}$ Test) was observed for plantar sensation; participants in G1 presented higher score of plantar sensation at Pre-test than Post and Follow-up tests (Table 2). For gait parameters, an interaction between factors (Group X Test) was observed for stride length. Participants in G1 showed greater stride length at Post-test compared to Pre-test (Table 2).

\section{Discussion}

This study observed benefits offered by textured insoles on plantar sensation and stride length in people with PD after continuous use. However, only plantar sensation benefits were kept after the follow-up period. Similar to studies with multiple sclerosis patients $[9,10]$, the continuous use of textured insoles was effective to increase stride length. Our results suggest that enhanced somatosensory feedback to the sensory system might result in an improved motor output of gait represented by the improvement of stride length - and might translate to a less hypometric pattern [3]. This finding is clinically relevant since the regulation of stride length is a key deficit of parkinsonian gait [11].

It is important to highlight that gait was assessed without the textured insoles. This suggests that wearing textured insoles in daily basis may provide a sensory-motor training effect (represented by the improvement of stride length that is still exhibited without the textured insoles), rather than a mechanical or cueing effect that would be dependent on having the insoles in the shoes at the time of testing [10]. Additionally, as the benefit on stride length was not maintained after the follow-up period, it might be speculated that longer wearing periods of the textured insoles are required to prolong the retention of benefits achieved.

The continuous use of textured insoles improved plantar sensation and the benefits were maintained after the follow-up period. Previous studies have indicated that short and long-term sensory stimulation may promote an expansion in the representation of the primary somatosensory cortex $[12,13]$. Thus, we speculate that the plantar stimulation may have contributed to an expansion in the representation of the primary somatosensory cortex and, as consequence, benefited plantar sensation. 
Table 2

Mean \pm standard deviation for pedometer, plantar sensation and gait variables and significant interactions between factors (Group X Test).

\begin{tabular}{|c|c|c|c|c|c|c|c|}
\hline \multirow[t]{2}{*}{ Variables } & \multicolumn{2}{|l|}{ Pre } & \multicolumn{2}{|l|}{ Post } & \multicolumn{2}{|l|}{ Follow up } & \multirow{2}{*}{$\frac{\text { Interactions }}{\text { (Group X Test) }}$} \\
\hline & G1 & G2 & G1 & G2 & G1 & G2 & \\
\hline $\begin{array}{l}\text { Pedometer (number of } \\
\text { steps) }\end{array}$ & - & - & $21139 \pm 13046$ & $23723 \pm 18624$ & $22633 \pm 15328$ & $31471 \pm 29863$ & ns \\
\hline $\begin{array}{l}\text { Plantar sensation (total } \\
\text { score) }\end{array}$ & $23.9 \pm 4.86$ & $21.6 \pm 3.40$ & $19.8 \pm 1.65^{*}$ & $21.2 \pm 3.07$ & $20.1 \pm 3.79 *$ & $21.1 \pm 3.87$ & $\begin{array}{l}\mathrm{F}_{2.34}=3.920 ; \mathrm{p}=0.029 \text { Post-hoc } \\
\left(\mathrm{p}=0.003^{* 11} ; \mathrm{p}=0,001^{* 2}\right)\end{array}$ \\
\hline Stride length (m) & $1.03 \pm 0.15$ & $1.11 \pm 0.07$ & $1.08 \pm 0.15^{*}$ & $1.09 \pm 0.12$ & $1.07 \pm 0.16$ & $1.10 \pm 0.12$ & $\begin{array}{l}\mathrm{F}_{2.34}=4.015 ; \mathrm{p}=0.027 \text { Post-hoc } \\
\left(\mathrm{p}=0.019^{* 1}\right)\end{array}$ \\
\hline Stride duration (s) & $1.06 \pm 0.12$ & $1.06 \pm 0.10$ & $1.01 \pm 0.09$ & $1.03 \pm 0.10$ & $1.03 \pm 0.13$ & $1.03 \pm 0.10$ & ns \\
\hline Velocity $(\mathrm{m} / \mathrm{s})$ & $0.99 \pm 0.19$ & $1.06 \pm 0.12$ & $1.08 \pm 0.19$ & $1.07 \pm 0.17$ & $1.06 \pm 0.22$ & $1.09 \pm 0.18$ & ns \\
\hline Cadence (strides/min) & $56.99 \pm 5.86$ & $57.15 \pm 5.63$ & $59.76 \pm 5.44$ & $58.86 \pm 5.82$ & $58.94 \pm 6.78$ & $58.92 \pm 5.54$ & ns \\
\hline Step width $(\mathrm{cm})$ & $14.67 \pm 3.65$ & $12.19 \pm 3.8$ & $14.19 \pm 3.03$ & $12.42 \pm 4.3$ & $14.44 \pm 3.13$ & $12.18 \pm 3.47$ & ns \\
\hline $\begin{array}{l}\text { Stance phase duration } \\
\text { (\%) }\end{array}$ & $37.02 \pm 4.15$ & $40.39 \pm 4.07$ & $34.62 \pm 3.18$ & $35.61 \pm 2.19$ & $35.37 \pm 3.66$ & $35.05 \pm 2.35$ & ns \\
\hline $\begin{array}{l}\text { Double-support } \\
\text { duration (\%) }\end{array}$ & $23.45 \pm 5.36$ & $23.07 \pm 3.98$ & $21.58 \pm 4.98$ & $21.26 \pm 2.61$ & $21.05 \pm 4.98$ & $21.46 \pm 2.93$ & ns \\
\hline
\end{tabular}

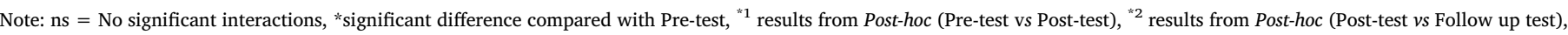
$\mathrm{G} 1$ = textured insole, $\mathrm{G} 2$ = control insole.

\subsection{Limitations and future directions}

The small sample size might have prevented us of finding other significant differences. However, this pilot study indicates the potential of textured insoles as intervention strategy for gait in people with PD. As we explored the continuous use of textured insole in short-term period (one week), future studies should verify if longer wearing periods might promote greater benefits or adaptation to the stimulus. Clinical trials with larger sample sizes are encouraged.

\section{Conclusion}

Continuous use of textured insoles (one week) benefits plantar sensation and gait in patients with PD. However, only the benefits in plantar sensation are maintained after a wash out period.

\section{Conflict of interest statement}

The authors have no conflicts of interest to disclose. All authors disclose any financial and personal relationships with other people or organizations that could inappropriately influence (bias) our work and manuscript. All authors have approved the final article.

\section{Acknowledgements}

The authors thank São Paulo Research Foundation (FAPESP) [grant \#2010/036500-8) for financial support and Podaly for manufacturing the insoles. The authors also acknowledge all members of the PROPARKI Group.

\section{References}

[1] B. Prätorius, S. Kimmeskamp, T.L. Milani, The sensitivity of the sole of the foot in patients with Morbus Parkinson, Neurosci. Lett. 346 (2003) 173-176.

[2] M. Alfuth, D. Rosenbaum, Effects of changes in plantar sensory feedback on human gait characteristics: a systematic review, Footwear Sci. 4 (2012) 1-22.

[3] M.E. Jenkins, Q.J. Almeida, S.J. Spaulding, R.B. Van Oostveen, J.D. Holmes, A.M. Johnson, S.D. Perry, Plantar cutaneous sensory stimulation improves singlelimb support time, and EMG activation patterns among individuals with Parkinson's disease, Parkinsonism Relat. Disord. 15 (2009) 697-702.

[4] E. Lirani-Silva, R. Vitório, F.A. Barbieri, A.M. Baptista, P.C.R. Santos, L.T.B. Gobbi, Different types of additional somatosensory information do not promote immediate benefits on gait in patients with Parkinson's disease and older adults, Motriz 21 (2015) 244-249.

[5] M.M. Hoehn, M.D. Yahr, Parkinsonism: onset, progression and mortality, Neurology 17 (1967) 573-581.

[6] S.M.D. Brucki, R. Nitrini, P. Caramelli, P.H.F. Bertolucci, I.H. Okamoto, Suggestions for utilization of the mini-mental state examination in Brazil, Arq. Neuro-Psiquiatr. 61 (2003) 777-781.

[7] S. Fahn, R. Elton, Members of the UPDRS. development committee. The Unified Parkinson's disease rating scale, in: S. Fahn, C.D. Marsden, D. Calne, M. Goldstein (Eds.), Recent Developments in Parkinson's Disease, Mcmellam Health Care Information, Florham Park NJ, 1987, pp. 153-164.

[8] J. Bell-krotoski, E. Tomancik, The repeatability of testing with Semmes-Weinstein monofilaments, J. Hand Surg. Am. 12 (1987) 155-161.

[9] M. Alfuth, Textured and stimulating insoles for balance and gait impairments in patients with multiple sclerosis and Parkinson's disease: a systematic review and meta-analysis, Gait Posture 51 (2017) 132-141.

[10] J. Dixon, A.L. Hatton, J. Robinson, H. Gamesby-Iyayi, D. Hodgson, K. Rome, R. Warnett, D.J. Martin, Effect of textured insoles on balance and gait in people with multiple sclerosis: an exploratory trial, Physiotherapy 100 (2014) 142-149.

[11] M.E. Morris, R. Iansek, T.A. Matyas, J.J. Summers, The pathogenesis of gait hypokinesia in Parkinson's disease, Brain 117 (1994) 1169-1181.

[12] C.W.U. WU, Peripheral somatosensory stimulation induced cortical plasticity and its clinical application on functional restoration in chronic stroke, Conf. Proc. IEEE Eng. Med. Biol. Soc. 5 (2005) 5230-5233.

[13] A. Pascual-Leone, F. Torres, Plasticity of the sensorimotor cortex representation of the reading finger in Braille readers, Brain 116 (1993) 39-52. 